Theorem 6. Let $M$ be a summand of a direct sum of modules of torsion free rank one. Then $S(e, M)=g(e, M)$, for any equivalence class $e$ of height sequences.

\title{
REFERENCE
}

1. R. B. Warfield, Jr., Classification theorems for p-groups and modules over a discrete valuation ring, Bull. Amer. Math. Soc. 78 (1972), 88-92. MR 45 \#378.

DEPARTMENT OF MATHEMATICS, KANSAS STATE UNIVERSITY, NANHATTAN, KANSAS 66506

Current address: Department of Mathematics, Benedict College, Columbia, South Carolina 29204

PROCEEDINGS OF THE

AMERICAN MATHEMATICAL SOCIETY

Volume 49, Number 1, May 1975

\section{THE NUMBER OF PROPER MINIMAL QUASIVARIETIES OF GROUPOIDS}

\section{A. SHAFAAT}

ABSTRACT. It is shown that if an algebra has more than one element, is freely generated in some variety by one element and has a cancellative endomorphism semigroup then it generates a minimal quasivariety. This is used to construct uncountably many minimal quasivarieties of groupoids that are not varieties.

A quasivariety [1] $\mathcal{K}$ of algebras will be called implicationally complete or minimal if $\mathcal{K}$ has exactly two subquasivarieties, namely, $\mathcal{K}$ itself and the class of all singleton (one element) algebras. By a proper quasivariety we mean a quasivariety which is not a variety.

In the case of semigroups there are countably infinitely many minimal quasivarieties, only one of which is proper [3]. For groupoids in general

Received by the editors February 5, 1973 and, in revised form, January 28, 1974. AMS (MOS) subject classifications (1970). Primary 08A15.

Key words and phrases. Implicationally complete or minimal quasivarieties, (universal) algebras, groupoids, free monogenic groupoid, endomorphism semigroup, cancellative, identity, implication, proper quasivariety, fully invariant congruences, words. 
there are [3] uncountably many minimal quasivarieties.

In this note we prove two theorems.

Theorem 1. There are continuum many proper minimal quasivarieties of groupoids.

Our proof of the above theorem uses the following result which is of considerable generality and of possible independent interest.

Theorem 2. Let $\mathbf{F}$ be a nonsingleton algebra freely generated by one element in some variety. Let the endomorphism semigroup End $(\mathbf{F})$ of $\mathbf{F}$ be cancellative. Then the quasivariety generated by $\mathbf{F}$ is implicationally com. plete.

Our proof of Theorem 1, with some modifications, carries through for some other types of algebras, e.g., commutative groupoids, algebras with at least one binary operation and so on. Theorem 1 and these related results may be compared with Kalicki [2] where similar results are proved for minimal varieties.

We now turn to the proofs of our theorems. For this we need some definitions and lemmas.

Let $W$ be the set of all words [1] in one variable $x$ and one binary operation + ; so that the "word groupoid" $(W,+)$ is the absolutely free monogenic groupoid. On $W$ we define another binary operation "e." by: $v \cdot w=v(w)$, where as usual $v(w)$ denotes the word obtained from $v(x)$ by substituting $w$ for $x$ in $v(x)$. [For example, $(x+x) \cdot(x+x)=((x+x)+(x+x))$.] We shall often write $v w$ for $v \cdot w$.

It is immediately seen that $(W, \circ)$ is a semigroup with identity $x$, that $(W, \cdot)$ is isomorphic to End $((W,+))$ and that "." distributes on the right over + . Moreover, as noted in $[4],(W,$.$) is free (in the variety of all monoids).$

Our first lemma, which we shall find very useful, concerns congruences over the algebra $(W,+, \circ)$.

Lemma 1. Let $\rho$ be a congruence over $\left(W,+,{ }^{\circ}\right)$ such that $(W, \cdot) / \rho$ is right cancelative (i.e., satisfies the implication $v u=w u \rightarrow v=w$ ). Let $v=$ $v(x), w=w(x)$ be any two words in W. Then:

$$
v \equiv w(\rho)
$$

if and only if the identity $\forall y(v(y)=w(y))$ holds in $(W,+) / \rho$;

$$
v \not w(\rho)
$$

if and only if the "negation" $\forall y(v(y) \neq w(y))$ holds in $(W,+) / \rho$. 
Proof. (1.1) If $v \equiv w(\rho)$ then $v u \equiv w u\left(\rho^{\prime}\right.$ for all $u \in W$. That is, $v(u) \equiv$ $w(u)(\rho)$ for all $u \in W$, which is precisely what it means to say that $\forall y(v(y)=$ $w(y))$ holds in $(W,+) / \rho$. Conversely, if $v(u) \equiv w(u)(\rho)$ for all $u \in W$ then $v$ $\equiv w(\rho)$ follows by taking $u=x$.

(1.2) Since $(W, o) / \rho$ is right cancellative, therefore $v \not \equiv w(\rho)$ implies $v(u) . \equiv w(u)(\rho)$ for all $u \in W$. Hence $\forall y(v(y) \neq w(y))$ holds in $(W,+) / \rho$. The converse follows by taking $y=x$ in the given negation.

Proof of Theorem 2. We prove the theorem for groupoids, the general case being similar.

It follows from the proof of Lemma 1 (1.1) that every congruence over $(W,+,$.$) is a fully invariant [1]$ congruence over $(W,+)$ and conversely. Also, for every congruence $\rho$ over $(W,+, \cdot)$ the semigroup $(W, \cdot) / \rho$ is isomorphic to End $((W,+) / \rho)$. In view of these two remarks Theorem 2 will be proved if we show that $(W,+) / \rho$ generates a minimal quasivariety for every congruence $\rho$ over $(W,+, \cdot)$ such that $(W, \cdot) / \rho$ is right cancellative. For this let $K_{\rho}$ be the quasivariety generated by such a $(W,+) / \rho$. Let $\mathcal{K}$ be a subquasivariety of $\mathcal{K}_{\rho}, \mathcal{K} \neq \mathcal{K}_{\rho}$. We show that $\mathcal{K}$ is trivial (i.e., contains only singleton groupoids).

Let

$$
p_{1}\left(y_{1}, \cdots, y_{n}\right)=q_{1}\left(y_{1}, \cdots, y_{n}\right) \& \cdots \& p_{m}\left(y_{1}, \cdots, y_{n}\right)=q_{m}\left(y_{1}, \cdots, y_{n}\right)
$$

$$
\rightarrow p\left(y_{1}, \cdots, y_{n}\right)=q\left(y_{1}, \cdots, y_{n}\right)
$$

be an implication of $\mathcal{K}$ which does not hold in $(W,+) / \rho$, where in writing (a) we have omitted universal quantifiers following a general practice. (Such an implication exists since $\left.\mathcal{K} \neq K_{\rho}\right)$. That (a) does not hold in $(W,+) / \rho$ means that there exist $u_{1}, \cdots, u_{n} \in W$ such that modulo $\rho$ we have

$$
\begin{gathered}
p_{1}\left(u_{1}, \cdots, u_{n}\right) \equiv q_{1}\left(u_{1}, \cdots, u_{n}\right), \\
p_{m}\left(u_{1}, \cdots, u_{n}\right) \doteq q_{m}\left(u_{1}, \cdots, u_{n}\right), \\
p\left(u_{1}, \cdots, u_{n}\right) \equiv q_{1}\left(u_{1}, \cdots, u_{n}\right) .
\end{gathered}
$$

By Lemma 1 this implies that the identities

$$
\begin{aligned}
p_{1}\left(u_{1}(y), \cdots, u_{n}(y)\right) & =q_{1}\left(u_{1}(y), \cdots, u_{n}(y)\right) \\
& \vdots \\
p_{m}\left(u_{1}(y), \cdots, u_{n}(y)\right) & =q_{m}\left(u_{1}(y), \cdots, u_{n}(y)\right)
\end{aligned},
$$

the negation

$$
p\left(u_{1}(y), \cdots, u_{n}(y)\right) \neq q\left(u_{1}(y), \cdots, u_{n}(y)\right),
$$

and therefore the implication 


$$
p\left(u_{1}(y), \cdots, u_{n}(y)\right)=q\left(u_{1}(y), \cdots, u_{n}(y)\right) \rightarrow y=z
$$

hold in $(W,+) / \rho$. Then (b) and (c) hold in all groupoids of $\mathcal{K}_{\rho}$ and hence of $\mathcal{K}$. Now (a), (b) and (c) imply the identity $y=z$ for $\mathcal{K}$. This completes our proof.

Remark 1. It follows from the above proof that in Theorem 2 the assumption of End $(F)$ being cancellative can be weakened to the assumption that End $(\mathbf{F})$ is left or right cancellative according as the product $F \stackrel{\phi}{\rightarrow} F \stackrel{\psi}{\rightarrow} F$ of two endomorphisms $\phi, \psi$ is written as $\psi \phi$ or $\phi \psi$.

Our next two general lemmas will be used in the proof of Theorem 1.

Semma 2. If $\rho, \theta$ are distinct congruences over $(W,+,$.$) such that$ $(W,.) / \rho$ and $(W,.) / \theta$ are cancellative, then the quasivarieties $\mathcal{K}_{\rho}, \mathcal{K}_{\theta}$, generated by $(W,+) / \rho,(W,+) / \theta$ respectively, intersect trivially.

Proof. Since $\rho \neq \theta$, we can assume, without loss of generality, that $v \equiv$ $w(\rho), v \equiv \equiv(\theta)$ for some $v, w \in W$. Then by Lemma $1 v(y)=w(y)$ is an identity of $\mathcal{K}_{\rho}$ and $v(y)=w(y) \rightarrow y=z$ is an implication of $\mathcal{K}_{\theta}$. Hence $y=z$ is an identity of $\mathcal{K}_{\rho} \cap K_{\theta}$ and the lemma is proved.

Lemma 3. Let $(L, \subseteq)$ be the partly ordered set of congruences $\rho$ over $(W,+,$.$) such that (W,.) / \rho$ is right cancellative and nonsingleton. If $\rho \in L$ is not a maximal element of $(L, \subseteq)$ then $\mathcal{K}_{\rho}$ is a proper quasivariety.

Proof. By assumption, there exists $\theta \in L, \rho \neq \theta, \rho \subseteq \theta$, such that $(W,+) / \theta$ is nonsingleton or $\mathcal{K}_{\theta}$ is nontrivial. If $\mathcal{K}_{\rho}$ is a variety then $(W,+) / \theta$, being a homomorphic image of $(W,+) / \rho$, belongs to $K_{\rho}$ and hence $K_{\theta} \subseteq K_{\rho}$. This contradicts Lemma 2 since $\mathcal{K}_{\rho} \cap K_{\theta}=K_{\theta}$ is nontrivial.

We now define some special congruences over $(W,+, \cdot)$ which we shall use to prove Theorem 1 .

Let $R \subseteq W$. Define $\bmod R$ to be the congruence over $(W,+, \cdot)$ generated by $\{\langle r, x\rangle ; r \in R\}$; so that $(W,+) / \bmod R$ is the monogenic free groupoid in the variety defined by the identities $\forall y(r(y)=y), r \in R$. The congruences $\bmod R$ are studied in some detail in [4]. Here we only need the following lemma which follows directly from Corollary 3.2, Lemma 3.6 and Theorem 4.1 of [4].

Lemma 4. Let $R, S \subseteq\left\{(x+x)^{n}+x ; n=1,2,3, \cdots\right\}$. Then:

(4.1) The monoid $(W, \cdot) / \bmod R$ is free (in the variety of all monoids).

(4.2) If $R \neq S$ then $\bmod R \neq \bmod S$.

In what follows $Q$ will always denote a subset of $\left\{(x+x)^{n}+x ; n=3\right.$, $4, \cdots\}$. By Lemma 4 (4.2), 


$$
(x+x)+x,(x+x)^{2}+x \not \equiv x(\bmod Q)
$$

and therefore $(W,+) / \bmod Q$ is nonsingleton. The fact that $(x+x)+x$, $(x+x)^{2}+x$ are excluded from $Q$ will be used in the proof of Theorem 1 below.

Let $W_{Q}$ denote the groupoid $(W,+) / \bmod Q$ and let $K_{Q}$ denote the quasivariety generated by $W_{Q}$. Note that $\mathcal{K}_{Q}$ is nontrivial.

Proof of Theorem 1. By Lemma 4 (4.1) and the proof of Theorem 2 it follows that $\mathcal{K}_{Q}$ is minimal. By Lemma 4 (4.2) and Lemma 2 we see that the function $Q \rightarrow K_{Q}$ is one-to-one. Theorem 1 will therefore be ptoved if we show that $\mathcal{K}_{Q}$ is proper. For this we use Lemma 3 and show that mod $Q$ is not maximal in $(L, \subseteq)$. Let

$$
R=Q \cup\left\{(x+x)^{2}+x\right\}, \quad S=Q \cup\left\{(x+x)+x,(x+x)^{2}+x\right\} .
$$

Then by Lemma 4 (4.2) $\bmod Q, \bmod R$ and $\bmod S$ are distinct; so that we have $\bmod Q \subset \bmod R \subset \bmod S$, where each inclusion is proper. It follows that mod $Q$ cannot be maximal in $\left(L, \subseteq^{\prime}\right.$, and our proof is complete.

\section{REFERENCES}

1. P. M. Cohn, Universal algebra, Harper and Row, New York, 1965. MR 31 \#224.

2. J. Kalicki, The number of equationally complete classes of equations, Nederl. Akad. Wetensch. Proc. Ser. A 58 = Indag. Math. 17 (1955), 660-662. MR 17, 571. $761-768$.

3. A. Shafaat, On implicational completeness, J. Canad. Math. Soc. 26 (1974),

4. - Unique factorization and Fermat's last theorem in groupoidal domains (prepublication copy).

DÉPARTEMENT DE MATHÉMATIQUE, UNIVERSITÉ LAVAL, QUÉBEC $10^{e}$, CANADA 